\title{
The growth of structure in interacting dark energy models
}

\author{
Gabriela Caldera-Cabra* * and Roy Maartent \\ Institute of Cosmology $\&$ Gravitation, University of Portsmouth, Portsmouth PO1 3FX, UK \\ Bjoern Malte Schaeferf \\ Astronomisches Recheninstitut, Zentrum für Astronomie, \\ Universität Heidelberg, Mönchhofstraße 12, 69120 Heidelberg, Germany
}

(Dated: June 24, 2009)

\begin{abstract}
If dark energy interacts with dark matter, there is a change in the background evolution of the universe, since the dark matter density no longer evolves as $a^{-3}$. In addition, the non-gravitational interaction affects the growth of structure. In principle, these changes allow us to detect and constrain an interaction in the dark sector. Here we investigate the growth factor and the weak lensing signal for a new class of interacting dark energy models. In these models, the interaction generalises the simple cases where one dark fluid decays into the other. In order to calculate the effect on structure formation, we perform a careful analysis of the perturbed interaction and its effect on peculiar velocities. Assuming a normalization to today's values of dark matter density and overdensity, the signal of the interaction is an enhancement (suppression) of both the growth factor and the lensing power, when the energy transfer in the background is from dark matter to dark energy (dark energy to dark matter).
\end{abstract}

\section{INTRODUCTION}

Advances in cosmological observations have led to strong evidence for non-baryonic cold dark matter and for a late-time acceleration of the universe, possibly driven by a dark energy field (see, e.g. [1, 2, 3$]$ ). Dark energy and dark matter are the dominant sources in the 'standard' model for the evolution of the universe. Both are considered essential missing pieces in the cosmic puzzle - and both are currently only detected via their gravitational effects. There could therefore be an interaction between them without violating current observational constraints. Furthermore, such an interaction could alleviate the 'coincidence' problem (why are the energy densities in the two components of the same order of magnitude today?). And interacting dark energy, by exerting a nongravitational 'drag' on dark matter, introduces new features to structure formation, including possibly a new bias [4] and a violation by dark matter of the weak equivalence principle [5, 6].

In order to pursue the possibility of interacting dark energy, we need to compute the effect of the interaction on the background expansion and on structure formation, and then to confront the results with data. The main problem is that there is no fundamental theory to guide us as to the form of an interaction. This problem is in fact subsidiary to a bigger problem - that there is currently no fundamental theory to underpin any model of dark energy, including non-interacting dark energy. The situation is somewhat similar to that in reheating of the universe after inflation: there is no fundamental theory

\footnotetext{
*Electronic address: Gaby.Calderacabral@port.ac.uk

$\dagger$ Electronic address: Roy.Maartens@port.ac.uk

${ }^{\ddagger}$ Electronic address: spirou@ita.uni-heidelberg.de
}

for the inflaton field, and no fundamental theory to guide us as to the form of the interactions between the inflaton and other fields during reheating. In this situation, we are forced to adopt phenomenological models to explore and narrow down the space of possibilities.

Various interacting dark energy models have been put forward (see, e.g. 7. 8, 9, 10, 11, 12, 13, 14, 15, 16, 17, 18, 19, 20, 21, 22, 23, 24, 25, 26, 27, 28]). All of these models are phenomenological. Some of them are constructed specifically for mathematical simplicity - for example, models in which the energy exchange rate is proportional to the Hubble rate. We consider models which are similar to simple models of reheating and of curvaton decay, i.e., where the energy exchange is in the form of a decay of one species into another: the energy exchange is linear in the energy density of the decaying species, and the decay rate is constant. Such models were introduced in [17, 22], where dark matter decays to dark energy. Here we follow 29] and generalize the energy exchange to a linear combination of dark sector energy densities, including as special cases the decay of dark matter to dark energy, and the decay of dark energy to dark matter.

We analyze the growth rate of structure and the weak lensing convergence, both of which are sensitive to interacting dark energy. In a previous work [30], we considered the case of dark matter decaying to dark energy. Here we generalize to include the case of dark energy decaying to dark matter. In [30] we did not analyze the peculiar velocity of dark matter, but rather deduced the density perturbation evolution using qualitative arguments. Here we perform a a careful analysis of the peculiar velocity, which confirms the qualitative arguments of [30] - but also allows us to deal with the new case of dark energy decay, where the peculiar velocity analysis is essential for deriving the correct density perturbation evolution. 
Following the typical approach in non-interacting dark energy models, we use the parametrization of the equation of state for the dark energy [31, 32],

$$
w(a)=w_{0}+w_{a}(1-a)
$$

In the background, a general coupling can be described by the continuity equations of cold dark matter (c) and dark energy (x),

$$
\begin{aligned}
\dot{\bar{\rho}}_{c}+3 H \bar{\rho}_{c} & =\bar{Q}_{c}, \\
\dot{\bar{\rho}}_{x}+3 H(1+w) \bar{\rho}_{x} & =\bar{Q}_{x}=-\bar{Q}_{c},
\end{aligned}
$$

where $w=\bar{P}_{x} / \bar{\rho}_{x}$, the background metric is $d s^{2}=-d t^{2}+$ $a^{2} d \vec{x}^{2}$, and $\bar{Q}_{c}\left(\bar{Q}_{x}\right)$ is the rate of energy density transfer to dark matter (dark energy). Therefore $\bar{Q}_{c}<0$ (> 0 ) implies that the direction of energy transfer is dark matter $\rightarrow$ dark energy (dark energy $\rightarrow$ dark matter).

In order to avoid stringent "fifth force" constraints, we assume that baryons (b) and photons $(\gamma)$ are not coupled to dark energy: $\bar{Q}_{b}=0=\bar{Q}_{\gamma}$. We also neglect radiation since we are focusing on structure formation in the late universe. The energy balance equation for fluid $A$ is

$$
\dot{\bar{\rho}}_{A}+3 H\left(1+w_{A}\right) \bar{\rho}_{A}=\bar{Q}_{A}
$$

with $w_{c}=w_{b}=0, w_{x}=w$ and $\bar{Q}_{c}=-\bar{Q}_{x} \neq 0=\bar{Q}_{b}$. The Friedmann equation is

$$
H^{2}=\frac{8 \pi G}{3}\left(\bar{\rho}_{c}+\bar{\rho}_{x}+\bar{\rho}_{b}\right) .
$$

\section{Background dynamics}

Once a form of $\bar{Q}_{c}$ is given, the background dynamics are fully determined by Eqs. (4) and (5), with $w$ given by Eq. (11). Here we use the new model [29]

$$
\bar{Q}_{c}=-\left(\Gamma_{c} \bar{\rho}_{c}+\Gamma_{x} \bar{\rho}_{x}\right)
$$

where $\Gamma_{A}$ are constant energy density transfer rates. The special cases of Eq. (6) are: decay of dark matter to dark energy, i.e., $\Gamma_{c}>0=\Gamma_{x}$, and decay of dark energy to dark matter, i.e., $\Gamma_{x}<0=\Gamma_{c}$. Models of the pure-decay type have been used in reheating after inflation [33], and to describe the decay of dark matter into radiation 34] or of a curvaton field into radiation [35].

A complete dynamical systems analysis for Eq. (6) is given in [29] for the case $w=$ const, i.e., $w_{a}=0$ in Eq. (11). In this paper we will consider variable $w$, and the particular cases when one of the transfer rates $\Gamma_{A}$ is zero. The special case previously considered in [22] corresponds to $\Gamma_{x}=0$, with $w_{a}=0$. As shown in [22], when $\Gamma_{c}>0$ and $w_{a}=0$, the dark energy density becomes negative at early times. The source of this problem is the rigidity of the assumption that $w$ is constant - the problem does not arise for quintessence models. It can also be avoided with variable $w$ for suitable choices of $w_{0}, w_{a}$ in Eq. (1), as shown in [36].
The case $\Gamma_{c}<0$ (with $w_{a}=0$ ) avoids negative dark energy density, but there is no attractor solution; by contrast, there is a late-time attractor when $\Gamma_{c}>0$ [29]. Equation (2) has an exact solution for $\Gamma_{x}=0$,

$$
\bar{\rho}_{c}=\bar{\rho}_{c 0} a^{-3} \exp \left[-\Gamma_{c}\left(t-t_{0}\right)\right],
$$

which shows that the dark matter density is always positive, regardless of the sign of $\Gamma_{c}$. For the special case $\Gamma_{c}=0=w_{a}$, the dark energy density is always positive, as can be seen from the exact solution of Eq. (3):

$$
\bar{\rho}_{x}=\bar{\rho}_{x 0} a^{-3(1+w)} \exp \left[\Gamma_{x}\left(t-t_{0}\right)\right] .
$$

\section{DENSITY AND VELOCITY PERTURBATION EQUATIONS}

Since we are interested in the late universe we can neglect anisotropic stress, and scalar perturbations of the flat metric are given, in Newtonian gauge, by

$$
d s^{2}=-(1+2 \phi) d t^{2}+a^{2}(1-2 \phi) d \vec{x}^{2} .
$$

The $A$-fluid four-velocity is

$$
u_{A}^{\mu}=\left(1-\phi, \partial^{i} v_{A}\right) \text {. }
$$

Choosing the energy frame for the total four-velocity $u^{\mu}$, the total velocity potential $v$ is defined by

$$
v \sum\left(\bar{\rho}_{A}+\bar{P}_{A}\right)=\sum\left(\bar{\rho}_{A}+\bar{P}_{A}\right) v_{A} .
$$

The covariant form of energy-momentum transfer is

$$
\nabla_{\nu} T_{A}^{\mu \nu}=Q_{A}^{\mu}, \quad Q_{c}^{\mu}=-Q_{x}^{\mu} \neq 0=Q_{b}^{\mu} .
$$

The energy-momentum transfer four-vector can be split relative to the total four-velocity as 22]

$$
Q_{A}^{\mu}=Q_{A} u^{\mu}+F_{A}^{\mu}, Q_{A}=\bar{Q}_{A}+\delta Q_{A}, u_{\mu} F_{A}^{\mu}=0,
$$

where $F_{A}^{\mu}$ is the momentum density transfer rate, relative to $u^{\mu}$. Then it follows that $F_{A}^{\mu}=\left(0, \partial^{i} f_{A}\right)$, where $f_{A}$ is a momentum transfer potential, and

$$
\begin{aligned}
Q_{0}^{A} & =-\left[\bar{Q}_{A}(1+\phi)+\delta Q_{A}\right], \\
Q_{i}^{A} & =a^{2} \partial_{i}\left(f_{A}+\bar{Q}_{A} v\right) .
\end{aligned}
$$

The general evolution equations for the dimensionless density perturbations $\delta_{A}=\delta \rho_{A} / \rho_{A}$ and the velocity perturbations $v_{A}$ are [22]:

$$
\begin{aligned}
& \dot{\delta}_{A}+3 H c_{s A}^{2} \delta_{A}-\left(1+w_{A}\right) \frac{k^{2}}{a} v_{A} \\
& -3 H\left[3 H\left(1+w_{A}\right)\left(c_{s A}^{2}-w_{A}\right)+\dot{w}_{A}\right] v_{A}-3\left(1+w_{A}\right) \dot{\phi} \\
& =\frac{\delta Q_{A}}{\bar{\rho}_{A}}+\frac{\bar{Q}_{A}}{\bar{\rho}_{A}}\left[\phi-\delta_{A}-3 a H\left(c_{s A}^{2}-w_{A}\right) v_{A}\right], \\
& \dot{v}_{A}+H\left(1-3 c_{s A}^{2}\right) v_{A}+\frac{c_{s A}^{2}}{a\left(1+w_{A}\right)} \delta_{A}+\frac{\phi}{a} \\
& =\frac{1}{\left(1+w_{A}\right) \bar{\rho}_{A}}\left\{\bar{Q}_{A}\left[v-\left(1+c_{s A}^{2}\right) v_{A}\right]+f_{A}\right\} .
\end{aligned}
$$


The relativistic Poisson equation is:

$$
\frac{k^{2} \phi}{a^{2}}=-3 H \dot{\phi}-3 H^{2} \phi-4 \pi G\left(\bar{\rho}_{c} \delta_{c}+\bar{\rho}_{b} \delta_{b}+\bar{\rho}_{x} \delta_{x}\right) \text {. }
$$

Structure formation takes place in the Newtonian regime, on spatial scales much smaller than the horizon radius, $a / k \ll H^{-1}$. Then the gravitational potential and its time derivative may be neglected relative to matter density fluctuations. This allows us to discard the $\phi$ terms in Eq. (16) and the first two terms on the right of Eq. (18). Dark energy fluctuations may be neglected since dark energy has a high sound speed, and does not cluster on sub-Hubble scales (we take the sound speed of dark energy to be that of a standard scalar field model, $\left.c_{s x}^{2}=1\right)$. In the Newtonian regime, the evolution equations for dark matter become:

$$
\begin{aligned}
\dot{\delta}_{c}-\frac{k^{2}}{a} v_{c} & =\frac{1}{\bar{\rho}_{c}}\left(\delta Q_{c}-\bar{Q}_{c} \delta_{c}\right), \\
\dot{v}_{c}+H v_{c}+\frac{1}{a} \phi & =\frac{1}{\bar{\rho}_{c}}\left[\bar{Q}_{c}\left(v-v_{c}\right)+f_{c}\right],
\end{aligned}
$$

while for baryons:

$$
\begin{aligned}
\dot{\delta}_{b}-\frac{k^{2}}{a} v_{b} & =0, \\
\dot{v}_{b}+H v_{b}+\frac{1}{a} \phi & =0 .
\end{aligned}
$$

The Poisson equation in the Newtonian limit and neglecting dark energy clustering is

$$
\frac{k^{2}}{a^{2}} \phi=-4 \pi G\left(\bar{\rho}_{c} \delta_{c}+\bar{\rho}_{b} \delta_{b}\right)
$$

\section{Dark sector energy-momentum transfer}

In order to analyze structure formation in interacting dark energy models, we need to specify a covariant form of the transfer four-vectors, $Q_{c}^{\mu}=-Q_{x}^{\mu}$, which recovers the energy transfer model of Eq. (6) in the background.

Firstly, following and generalizing [22], we can promote Eq. (6) to the perturbed universe directly via

$$
Q_{c}:=\bar{Q}_{c}+\delta Q_{c}=-\Gamma_{c} \bar{\rho}_{c}\left(1+\delta_{c}\right)-\Gamma_{x} \bar{\rho}_{x}\left(1+\delta_{x}\right) .
$$

However, the background model provides no guide for specifying the momentum transfer (which vanishes in the background). It is crucial to provide a covariant prescription that fixes the momentum transfer, a point that is not always clearly recognised in the literature. Following [22], we specify the momentum transfer by the covariant physical requirement that there is no momentum transfer in the dark matter frame, i.e.,

$$
Q_{c}^{\mu}=Q_{c} u_{c}^{\mu} .
$$

Then Eqs. (24) and (25) completely describe the interaction.
Regardless of the specific form of $Q_{c}$, it follows from Eq. (25), using Eqs. (14) and (15), that

$$
f_{c}=\bar{Q}_{c}\left(v_{c}-v\right)
$$

so that Eq. (20) becomes

$$
\dot{v}_{c}+H v_{c}+\frac{\phi}{a}=0 \text {. }
$$

This means that the dark matter velocity is the same as the baryon velocity, and is not directly affected by the interaction with dark energy. In particular, the form of energy-momentum transfer in Eq. (25), for any $Q_{c}$, ensures that there is no violation of the weak equivalence principle for dark matter, as shown in [6]. This will not be true for interaction models where $Q_{c}^{\mu}$ is not parallel to $u_{c}^{\mu}$.

\section{GROWTH FACTOR AND WEAK LENSING: THE CASE $\Gamma_{x}=0$}

For this model,

$$
Q_{c}^{\mu}=-\Gamma_{c} \bar{\rho}_{c}\left(1+\delta_{c}\right) u_{c}^{\mu} .
$$

It follows from Eqs. (2) and (3) that in the background, $\Gamma_{c}>0$ corresponds to the decay of dark matter into dark energy, while $\Gamma_{c}<0$ describes energy transfer from dark energy to dark matter.

The equations for density and velocity perturbations and the Poisson equation are

$$
\begin{aligned}
\dot{\delta}_{c}-\frac{k^{2} v_{c}}{a} & =0, \\
\dot{v}_{c}+H v_{c}+\frac{\phi}{a} & =0, \\
k^{2} \phi & =-4 \pi G a^{2}\left(\bar{\rho}_{c} \delta_{c}+\bar{\rho}_{b} \delta_{b}\right) .
\end{aligned}
$$

From these equations, the second-order evolution equation for $\delta_{c}$ is

$$
\ddot{\delta}_{c}+2 H \dot{\delta}_{c}-4 \pi G\left(\bar{\rho}_{c} \delta_{c}+\bar{\rho}_{b} \delta_{b}\right)=0 .
$$

These equations have precisely the same form as those for the non-interacting case. However, the solutions $\delta_{c}$ are different because the background terms $H$ and $\bar{\rho}_{c}$ evolve differently. The interaction will produce a signal in the growth of structure, as measured by the growth factor and weak lensing measurements. Equation (32) confirms the qualitative arguments used in [30], where the velocity perturbations were not analyzed. In this section, we extend the analysis of [30] to include the case $\Gamma_{c}<0$.

For our computations, the initial conditions are set at the present epoch $a_{0}=1$, and we integrate backwards to $a=10^{-2}$. We normalize the dark matter background densities and density perturbations in the interacting and non-interacting cases to today's values, $\Omega_{c 0}$ and $\delta_{c 0}$. We 
take $\Gamma_{c}= \pm 0.3 H_{0}$, and use an equation of state (11) with $w_{0}=-0.99$ and $w_{a}=0.8$. These values are known [36] to avoid $\bar{\rho}_{x}<0$ in the past when $\Gamma_{c}>0$. For simplicity, and since we are only illustrating the effects of interacting dark energy, rather than making accurate parameter estimations, we will neglect the baryons in our computations.

In Fig. 1 we plot the energy densities $\Omega_{A}=$ $8 \pi G \bar{\rho}_{A} / 3 H^{2}$. Models with dark matter decay $\left(\Gamma_{c}>0\right)$ have higher dark matter density in the past relative to the non-interacting models (recall that we normalize the densities in the two models to the same values at $a_{0}=1$ ). By contrast, models with energy transfer from dark energy to dark matter $\left(\Gamma_{c}<0\right)$ exhibit lower dark matter density in the past.

Figure 2 shows the growth function $D_{+}=\delta_{c} / \delta_{c 0}$ in the interacting models compared to the non-interacting case, normalized to today's value. The quantity $D_{+} / a$ reflects the time evolution of the gravitational potential $\phi$. The model with decaying dark matter, $\Gamma_{c}>0$, shows an enhancement of structure growth relative to the non-interacting model, since a lower initial value at early times leads to the same final value today, $\delta_{c 0}$, as the noninteracting model. By contrast, models with $\Gamma_{c}<0$ show a suppression of structure growth. These features are consistent with the background evolution shown in Fig. 1 - recall that the difference in the growth function is determined entirely by the different background evolution, since Eq. (32) has the same form as the non-interacting version. When $\Gamma_{c}>0$, there is more dark matter in the past, and this leads to an enhancement in the growth of structure. The reverse holds for $\Gamma_{c}<0$. Note that the enhancement / suppression is specific to the circumstance that the interacting and non-interacting models are normalized to have the same parameters today, i.e., $\Omega_{c 0}$ and $\delta_{c 0}$.

The weak lensing convergence spectrum is given by [30]

$$
C_{k}(\ell)=\frac{9}{4 c^{4}} \int_{0}^{\chi} d \chi G^{2}(a H)^{4} \Omega_{c}^{2} D_{+}^{2} P(k=\ell / \chi),
$$

where $\chi(a)=\int_{a}^{1} d a / a^{2} H(a)$ is the comoving distance, $P(k)$ is the dark matter power spectrum, and

$$
G(\chi)=\int_{0}^{\chi} d \chi^{\prime} p(z) \frac{d z}{d \chi^{\prime}} \frac{\chi^{\prime}-\chi}{\chi^{\prime}}
$$

with $p(z)$ giving the redshift distribution of lensing galaxies.

Figure 3 illustrates the impact of interacting dark energy on the weak lensing convergence power spectra. The lensing power in models with decaying dark matter $\left(\Gamma_{c}>0\right)$ is higher since the dark matter density in the past was higher (see Fig. 1), compared to non-interacting models - assuming that the models are normalized to have the same values today of $\Omega_{c 0}$. The higher matter density leads to stronger gravitational potential and hence a stronger light deflection. When $\Gamma_{c}<0$, the reverse situation applies.
Our results in the case $\Gamma_{c}>0$ are consistent with those of [30], where in addition the weak lensing bispectrum is computed. The case $\Gamma_{c}<0$ was not considered in [30].

\section{GROWTH FACTOR AND WEAK LENSING: THE CASE $\Gamma_{c}=0$}

Weak lensing has not previously been analyzed in this model, for which

$$
Q_{c}^{\mu}=-\Gamma_{x} \bar{\rho}_{x}\left(1+\delta_{x}\right) u_{c}^{\mu} .
$$

It follows from Eqs. (2) and (3) that in the background, $\Gamma_{x}<0$ corresponds to the decay of dark energy into dark matter, while $\Gamma_{x}>0$ describes energy transfer from dark matter to dark energy. This model does not present the problem of negative dark energy density in the past.

The velocity perturbation equation (27) is the same as in the previous model, but the density perturbation equation (19) has a source term:

$$
\dot{\delta}_{c}-\frac{k^{2} v_{c}}{a}=\Gamma_{x} \frac{\bar{\rho}_{x}}{\bar{\rho}_{c}} \delta_{c}
$$

which will generate a linear bias between dark matter and baryons. This source term leads to a modified evolution equation for $\delta_{c}$ :

$$
\begin{aligned}
& \ddot{\delta}_{c}+2 H\left(1-\frac{\Gamma_{x}}{H} \frac{\bar{\rho}_{x}}{\bar{\rho}_{c}}\right) \dot{\delta}_{c}-4 \pi G\left\{\overline { \rho } _ { c } \left[1+\frac{2}{3 a} \frac{\Gamma_{x}}{H} \frac{\bar{\rho}_{x}}{\bar{\rho}_{c}} \times\right.\right. \\
& \left.\left.\times\left\{2-3 w+\frac{\Gamma_{x}}{H}\left(1+\frac{\bar{\rho}_{x}}{\bar{\rho}_{c}}\right)\right\}\right] \delta_{c}+\bar{\rho}_{b} \delta_{b}\right\}=0 .
\end{aligned}
$$

The evolution equation for dark matter density perturbations is modified relative to the non-interacting models in 3 ways:

(1) due to the different background evolution (affecting the terms $\left.\bar{\rho}_{c}, \bar{\rho}_{x}, H\right)$;

(2) due to the modified Hubble friction term, $H \rightarrow$ $H\left(1-\Gamma_{x} \bar{\rho}_{x} / H \bar{\rho}_{c}\right)$

(3) due to the modified source term, which gives rise to a modified effective Newton constant $G_{\text {eff }}$ for dark matter:

$$
\frac{G_{\mathrm{eff}}}{G}=1+\frac{2}{3 a} \frac{\Gamma_{x}}{H} \frac{\bar{\rho}_{x}}{\bar{\rho}_{c}}\left\{2-3 w+\frac{\Gamma_{x}}{H}\left(1+\frac{\bar{\rho}_{x}}{\bar{\rho}_{c}}\right)\right\},
$$

so that

$$
\ddot{\delta}_{c}+2 H\left(1-\frac{\Gamma_{x}}{H} \frac{\bar{\rho}_{x}}{\bar{\rho}_{c}}\right) \dot{\delta}_{c}-4 \pi G_{\mathrm{eff}} \bar{\rho}_{c} \delta_{c}-4 \pi G \bar{\rho}_{b} \delta_{b}=0
$$

Compared to the previous model, where only modification (1) operates, this model shows a more complicated change from the non-interacting case. 

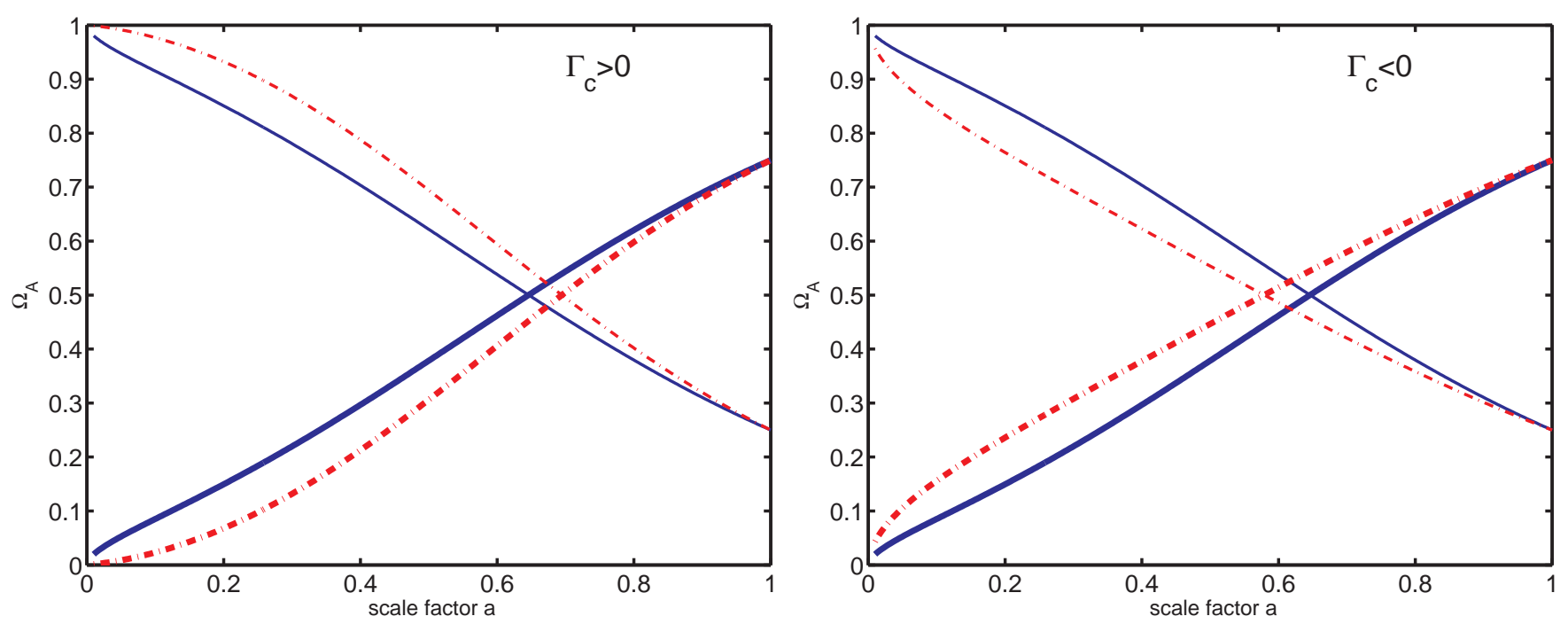

FIG. 1: The densities of dark matter, $\Omega_{c}$ (thin lines), and dark energy, $\Omega_{x}$ (thick lines), in the interacting models (dashed-dotted lines), with $\Gamma_{c}= \pm 0.3 H_{0}$, and non-interacting models (solid lines), normalized to today's values.
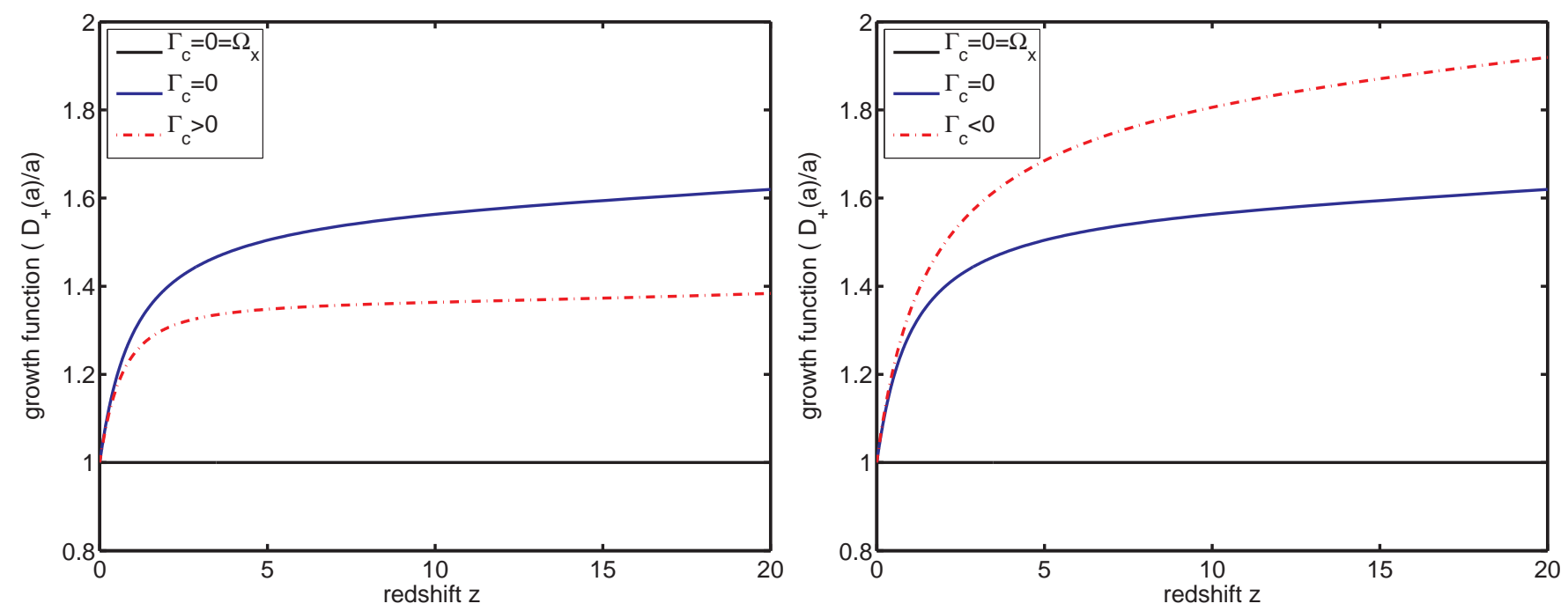

FIG. 2: Linear growth function $D_{+}=\delta_{c} / \delta_{c 0}$, normalized to today's value, relative to its value in a pure-matter model $\left(D_{+}=a\right)$. The interacting models (dashed-dotted lines), with $\Gamma_{c}= \pm 0.3 H_{0}$, are shown in comparison to non-interacting models (solid lines).

Figure 4 shows the energy densities $\Omega_{A}$. (We again neglect baryons in the plots.) The model with energy transfer from dark matter to dark energy $\left(\Gamma_{x}>0\right)$, has higher dark matter density in the past. The model with dark energy decay to dark matter $\left(\Gamma_{x}<0\right)$ has lower dark matter density in the past. (We normalize to today's value of $\Omega_{c 0}$.)

In Fig. 5 we show the growth function obtained by solving Eq. (39) (neglecting baryons). As in the previous model, we see that an energy transfer from dark matter to dark energy, $\Gamma_{x}>0$, leads to enhancement of growth, since less initial power is needed to obtain the same final value as the non-interacting model. The reverse holds for the model with $\Gamma_{x}<0$, which suppresses growth for the same final value $\delta_{c 0}$. These background effects are reinforced by the modifications (2) and (3) to the $\delta_{c}$ evolution equation Eq. (39):

- When $\Gamma_{x}>0$, the Hubble friction term is reduced, and the source term is enhanced, both contributing to an increase in $\delta_{c}$ relative to the non-interacting case.

- For $\Gamma_{x}<0$, the friction term is enhanced and the source term is reduced, both contributing to a reduction in $\delta_{c}$. 

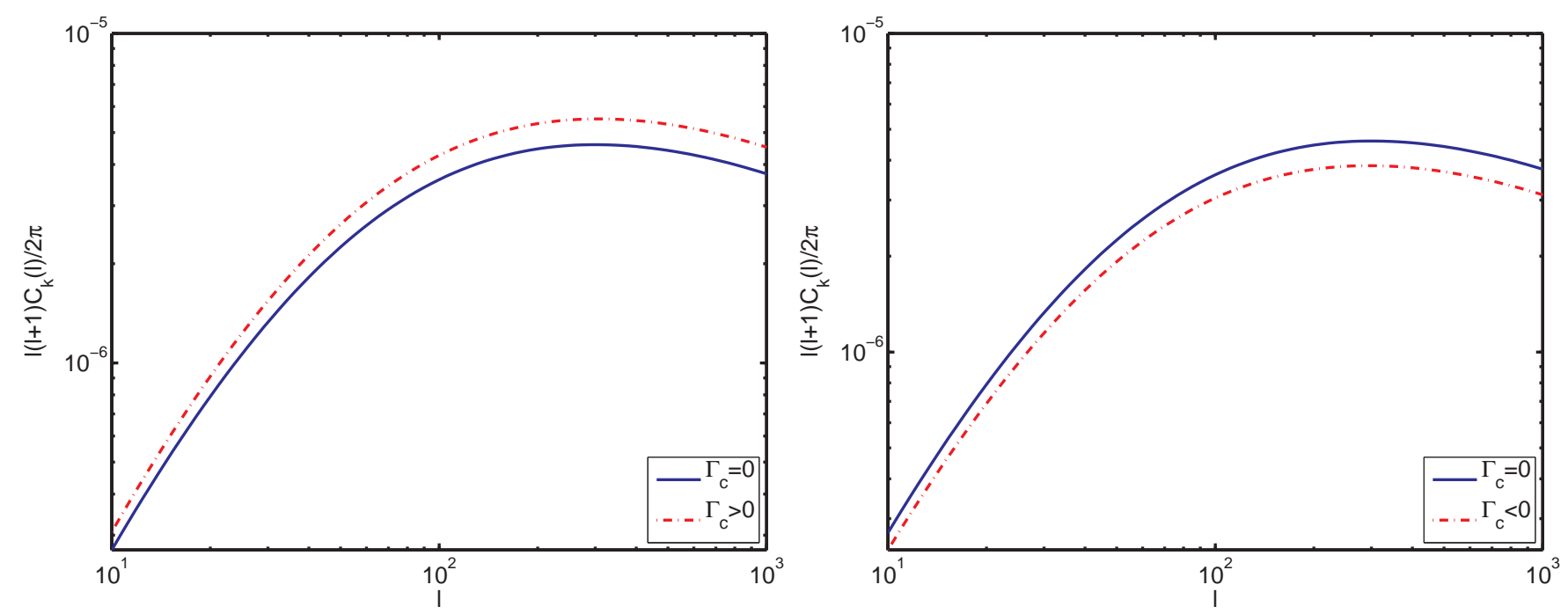

FIG. 3: Weak lensing convergence power spectra $\ell(\ell+1) C_{k}(\ell) / 2 \pi$ in the interacting models (dashed-dotted lines), with $\Gamma_{c}= \pm 0.3 H_{0}$, in comparison to non-interacting models (solid lines).
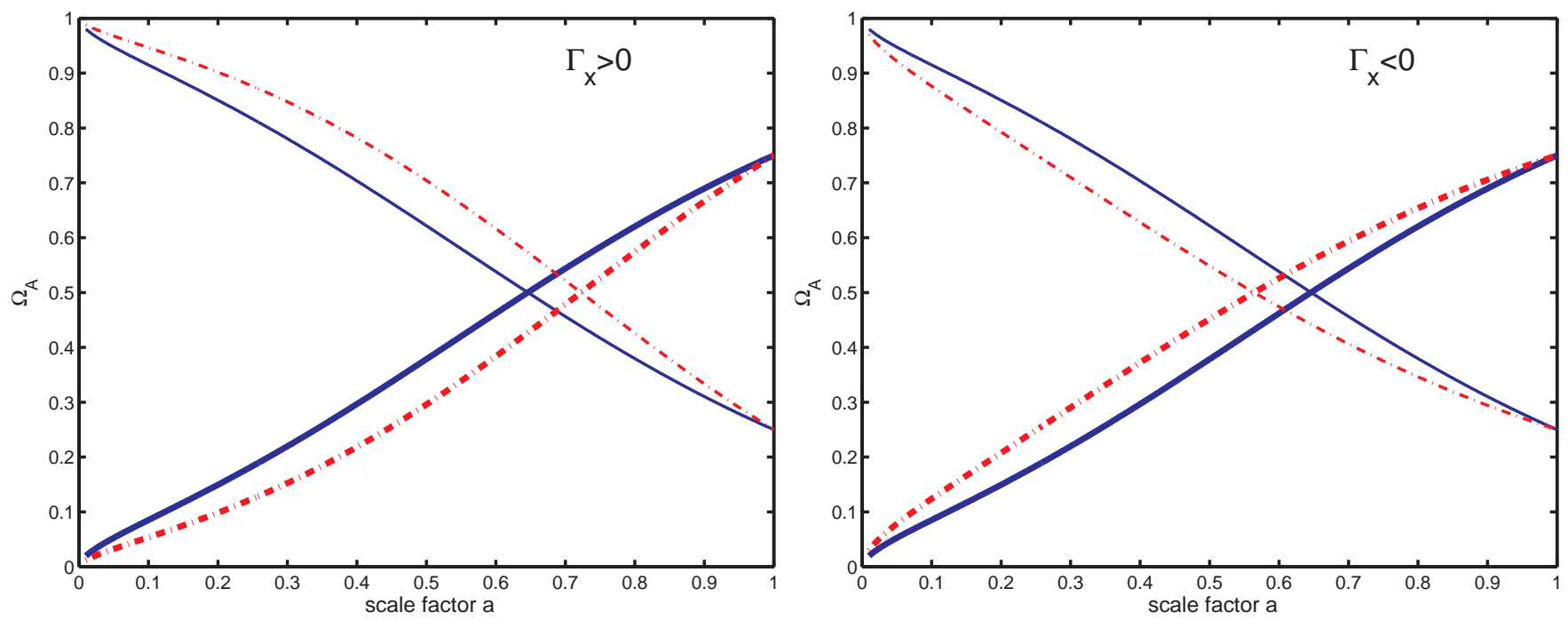

FIG. 4: The densities of dark matter, $\Omega_{c}$ (thin lines), and dark energy, $\Omega_{x}$ (thick lines), in the interacting models (dashed-dotted lines), with $\Gamma_{x}=+0.3 H_{0},-0.2 H_{0}$, and non-interacting models (solid lines), normalized to today's values.

Using the growth function in Eq. (33), we compute the impact of interacting dark energy on the weak lensing convergence power spectra. The results are shown in Fig. 6. given the normalization to today's values of $\Omega_{c 0}$ and $\delta_{c 0}$, the model with $\Gamma_{x}>0\left(\Gamma_{x}<0\right)$ exhibits enhanced (suppressed) power relative to the non-interacting model. The cause of this is both the background effect, i.e., since the matter density in the past was higher (lower) than the non-interacting case, and the additional reinforcing effects of modified Hubble friction and modified Newton constant. These effects work together to produce stronger (weaker) gravitational potentials and hence a stronger (weaker) light deflection.

\section{CONCLUSIONS}

For the interacting dark energy models described by the covariant interaction four-vector

$$
\begin{aligned}
Q_{c}^{\mu} & =Q_{c} u_{c}^{\mu}, \\
Q_{c} & =-\Gamma_{c} \bar{\rho}_{c}\left(1+\delta_{c}\right)-\Gamma_{x} \bar{\rho}_{x}\left(1+\delta_{x}\right),
\end{aligned}
$$

we investigated the effect of the interaction on the background evolution of densities, on the growth factor in structure formation, and on the weak lensing convergence power spectrum.

We considered the two cases, (I) $\Gamma_{x}=0$ and (II) $\Gamma_{c}=$ 0 separately. In the general case where both transfer rates are nonzero, the results are a linear superposition 

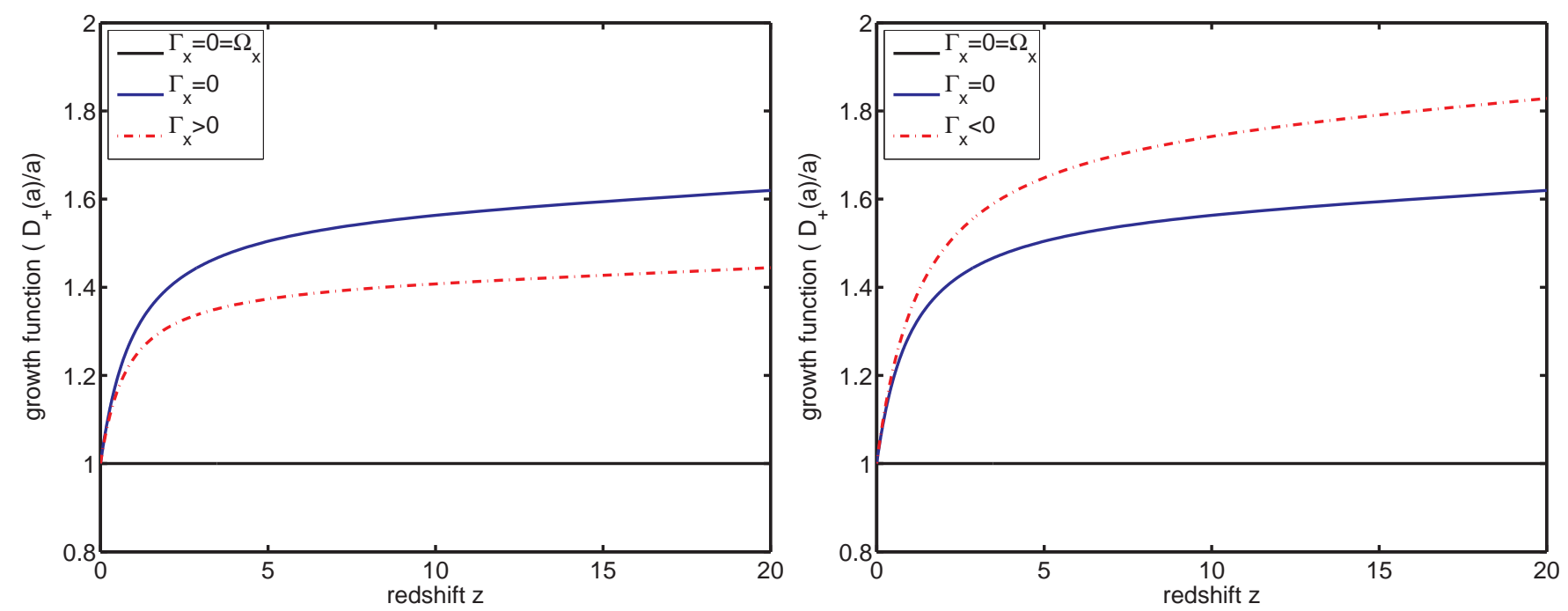

FIG. 5: Linear growth function $D_{+}=\delta_{c} / \delta_{c 0}$, normalized to today's value, relative to its value in a pure-matter model $\left(D_{+}=a\right)$. The interacting models (dashed-dotted lines), with $\Gamma_{x}=+0.3 H_{0},-0.2 H_{0}$, are shown in comparison to non-interacting models (solid lines).
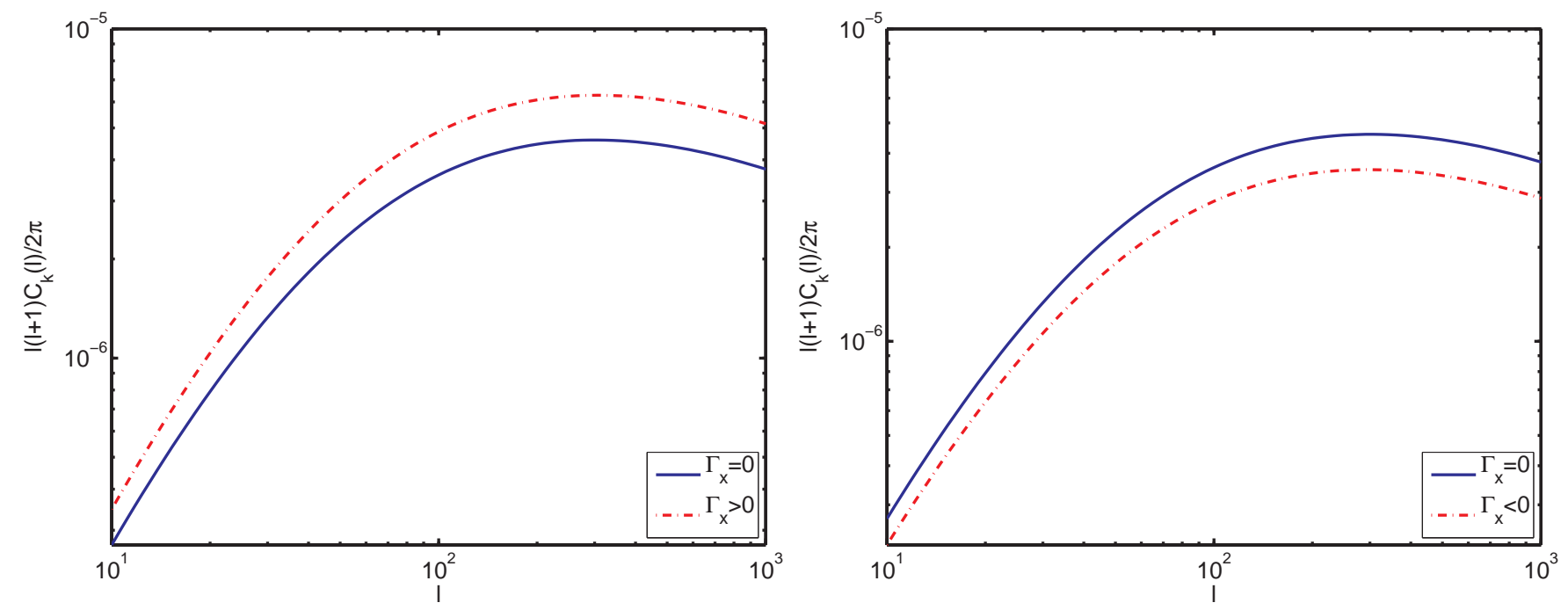

FIG. 6: Weak lensing convergence power spectra $\ell(\ell+1) C_{k}(\ell) / 2 \pi$ in the interacting models (dashed-dotted lines), with $\Gamma_{x}=+0.3 H_{0},-0.2 H_{0}$, in comparison to non-interacting models (solid lines).

of the two cases, and will depend on the signs and relative magnitudes of $\Gamma_{A}$ [the density perturbations will be given by Eq. [39] ].

Case I does not produce changes in the evolution equation for the dark matter density perturbations, so that the change in the growth factor and weak lensing is due purely to the different background evolution. In case II, the $\delta_{c}$ evolution equation has two additional modifications: a modified Hubble friction term, and a modified source term, expressed as a modified effective Newton constant for dark matter:

$$
\ddot{\delta}_{c}+2 H\left(1-\frac{\Gamma_{x}}{H} \frac{\bar{\rho}_{x}}{\bar{\rho}_{c}}\right) \dot{\delta}_{c}-4 \pi G_{\mathrm{eff}} \bar{\rho}_{c} \delta_{c}-4 \pi G \bar{\rho}_{b} \delta_{b}=0,
$$

where

$$
\frac{G_{\text {eff }}}{G}=1+\frac{2}{3 a} \frac{\Gamma_{x}}{H} \frac{\bar{\rho}_{x}}{\bar{\rho}_{c}}\left\{2-3 w+\frac{\Gamma_{x}}{H}\left(1+\frac{\bar{\rho}_{x}}{\bar{\rho}_{c}}\right)\right\} .
$$

These modifications act in the same direction as the modification to the background evolution.

Both cases present the same qualitative feature in the background evolution and in the growth of structure, assuming a normalization to today's values of $\Omega_{c 0}$ and $\delta_{c 0}$ : for energy transfer from dark matter to dark energy, i.e. $\Gamma_{A}>0$, the dark matter density is higher in the past relative to the non-interacting case, and hence the gravitational potential is higher, leading to an enhancement of growth and a stronger lensing signal. By contrast, when 
the energy transfer is from dark energy to dark matter, $\Gamma_{A}<0$, the dark matter density is lower in the past, leading to a suppression of growth and a reduced lensing signal. In the case $\Gamma_{c}=0$, the effects of the background density evolution are reinforced by the modifications to the Hubble friction and source terms in the density perturbation equation (42).

Our conclusions are subject to some caveats:

- The enhancement or suppression of growth and lensing power relative to the non-interacting case is tied to the assumption that the current values of $\Omega_{c 0}$ and $\delta_{c 0}$ are equal in the two cases. This gives an accurate picture of how the effects of the interaction operate - but we cannot conclude that there is relative enhancement or suppression, since in reality the current values of $\Omega_{c 0}$ and $\delta_{c 0}$ will not typically be equal. The best-fit values, based on a range of observations, are likely to be different, as shown for other interaction models in [18, 24, 27, 37, 38, 39, 40] and for the $\Gamma_{x}=0$ model in 36].

- We have followed the standard practice of imposing the Newtonian approximation on sub-Hubble scales: for scales close to the Hubble radius, correction terms would start to appear, and numerical integration of the exact perturbation equations (16)(18) would be necessary.

- The weak lensing signal that we have computed is strictly only valid on linear scales. On nonlin- ear scales, the effects of the interaction would need to be computed (see, e.g. [41, 42, 43, 44, 45]) in order to construct an accurate lensing signal, and this could involve N-body simulations for interacting dark matter [46, 47, 48].

Finally, we note that the integrated Sachs-Wolfe effect will also carry an imprint of the dark sector interaction, and this is investigated for the $\Gamma_{x}=0$ model in [36, 49], and for a different interaction model in [50].

\section{Acknowledgments}

GC-C is supported by the Programme Alban (the European Union Programme of High Level Scholarships for Latin America), scholarship No. E06D103604MX, and the Mexican National Council for Science and Technology, CONACYT, scholarship No. 192680. The work of RM was supported by the UK's Science \& Technology Facilities Council. The work of BMS is supported by the German Research Foundation (DFG) within the framework of the excellence initiative through the Heidelberg Graduate School of Fundamental Physics. RM thanks the Cosmology Group at the University of Cape Town, where part of this work was done, supported by a joint Royal Society (UK) and National Research Foundation (South Africa, UID65329) exchange grant on Dark Energy. We thank Kazuya Koyama and Yong-seon Song for helpful discussions. GC-C thanks Jim Cresswell and Kelly Nock for very helpful advice on numerical issues.
[1] J. Dunkley et al. (WMAP), Astrophys. J. Suppl. 180, 306 (2009), 0803.0586.

[2] M. Tegmark et al. (SDSS), Phys. Rev. D74, 123507 (2006), astro-ph/0608632.

[3] W. J. Percival et al., Mon. Not. Roy. Astron. Soc. 381, 1053 (2007), 0705.3323.

[4] L. Amendola and D. Tocchini-Valentini, Phys. Rev. D66, 043528 (2002), astro-ph/0111535.

[5] O. Bertolami, F. Gil Pedro, and M. Le Delliou, Phys. Lett. B654, 165 (2007), astro-ph/0703462.

[6] K. Koyama, R. Maartens, and Y.-S. Song (2009).

[7] C. Wetterich, Astron. Astrophys. 301, 321 (1995), hepth/9408025.

[8] L. Amendola, Phys. Rev. D60, 043501 (1999), astro$\mathrm{ph} / 9904120$.

[9] A. P. Billyard and A. A. Coley, Phys. Rev. D61, 083503 (2000), astro-ph/9908224.

[10] W. Zimdahl and D. Pavon, Phys. Lett. B521, 133 (2001), astro-ph/0105479.

[11] G. R. Farrar and P. J. E. Peebles, Astrophys. J. 604, 1 (2004), astro-ph/0307316.

[12] L. P. Chimento, A. S. Jakubi, D. Pavon, and W. Zimdahl, Phys. Rev. D67, 083513 (2003), astro-ph/0303145.

[13] G. Olivares, F. Atrio-Barandela, and D. Pavon, Phys. Rev. D71, 063523 (2005), astro-ph/0503242.
[14] T. Koivisto, Phys. Rev. D72, 043516 (2005), astro$\mathrm{ph} / 0504571$.

[15] H. M. Sadjadi and M. Alimohammadi, Phys. Rev. D74, 103007 (2006), gr-qc/0610080.

[16] Z.-K. Guo, N. Ohta, and S. Tsujikawa, Phys. Rev. D76, 023508 (2007), astro-ph/0702015.

[17] C. G. Boehmer, G. Caldera-Cabral, R. Lazkoz, and R. Maartens, Phys. Rev. D78, 023505 (2008), 0801.1565.

[18] J.-H. He and B. Wang, JCAP 0806, 010 (2008), 0801.4233 .

[19] M. Quartin, M. O. Calvao, S. E. Joras, R. R. R. Reis, and I. Waga, JCAP 0805, 007 (2008), 0802.0546.

[20] S. H. Pereira and J. F. Jesus, Phys. Rev. D79, 043517 (2009), 0811.0099.

[21] C. Quercellini, M. Bruni, A. Balbi, and D. Pietrobon (2008), 0803.1976.

[22] J. Valiviita, E. Majerotto, and R. Maartens, JCAP 0807, 020 (2008), 0804.0232.

[23] J.-H. He, B. Wang, and E. Abdalla (2008), 0807.3471.

[24] R. Bean, E. E. Flanagan, I. Laszlo, and M. Trodden, Phys. Rev. D78, 123514 (2008), 0808.1105.

[25] S. Chongchitnan, Phys. Rev. D79, 043522 (2009), 0810.5411.

[26] P. S. Corasaniti, Phys. Rev. D78, 083538 (2008), 0808.1646 . 
[27] M. B. Gavela, D. Hernandez, L. L. Honorez, O. Mena, and S. Rigolin (2009), 0901.1611.

[28] B. M. Jackson, A. Taylor, and A. Berera, Phys. Rev. D79, 043526 (2009), 0901.3272.

[29] G. Caldera-Cabral, R. Maartens, and L. A. Urena-Lopez, Phys. Rev. D79, 063518 (2009), 0812.1827.

[30] B. M. Schaefer, G. A. Caldera-Cabral, and R. Maartens (2008), 0803.2154.

[31] M. Chevallier and D. Polarski, Int. J. Mod. Phys. D10, 213 (2001), gr-qc/0009008.

[32] E. V. Linder and A. Jenkins, Mon. Not. Roy. Astron. Soc. 346, 573 (2003), astro-ph/0305286.

[33] M. S. Turner, Phys. Rev. D28, 1243 (1983).

[34] R. Cen (2000), astro-ph/0005206.

[35] K. A. Malik, D. Wands, and C. Ungarelli, Phys. Rev. D67, 063516 (2003), astro-ph/0211602.

[36] J. Valiviita, R. Maartens, and E. Majerotto (2009).

[37] S. Lee, G.-C. Liu, and K.-W. Ng, Phys. Rev. D73, 083516 (2006), astro-ph/0601333.

[38] G. La Vacca and L. P. L. Colombo, JCAP 0804, 007 (2008), 0803.1640.

[39] L. Vergani, L. P. L. Colombo, G. La Vacca, and S. A. Bonometto, Astrophys. J. 697, 1946 (2009), 0804.0285.
[40] G. La Vacca, J. R. Kristiansen, L. P. L. Colombo, R. Mainini, and S. A. Bonometto, JCAP 0904, 007 (2009), 0902.2711.

[41] M. Manera and D. F. Mota, Mon. Not. Roy. Astron. Soc. 371, 1373 (2006), astro-ph/0504519.

[42] R. Mainini and S. Bonometto, Phys. Rev. D74, 043504 (2006), astro-ph/0605621.

[43] E. Abdalla, L. R. W. Abramo, J. Sodre, L., and B. Wang, Phys. Lett. B673, 107 (2009), 0710.1198.

[44] P. M. Sutter and P. M. Ricker (2008), 0804.4172.

[45] R. Mainini, JCAP 0904, 017 (2009), 0903.0574.

[46] A. V. Maccio, C. Quercellini, R. Mainini, L. Amendola, and S. A. Bonometto, Phys. Rev. D69, 123516 (2004), astro-ph/0309671.

[47] M. Baldi, V. Pettorino, G. Robbers, and V. Springel (2008), 0812.3901.

[48] V. Pettorino and C. Baccigalupi, Phys. Rev. D77, 103003 (2008), 0802.1086.

[49] B. M. Schaefer (2008), 0803.2239.

[50] G. Olivares, F. Atrio-Barandela, and D. Pavon, Phys. Rev. D77, 103520 (2008), 0801.4517. 